\title{
Mature Teratoma of the Petrous Bone with Extension into the Cerebellopontine Angle: Case Report
}

\author{
Nickalus Khan ${ }^{1}$ Paul Klimo Jr. ${ }^{1,2,3,4}$ Julie Harreld ${ }^{5}$ \\ ${ }^{1}$ Department of Neurosurgery, University of Tennessee Health \\ Science Center, Memphis, Tennessee, United States \\ 2 Semmes-Murphey Neurologic \& Spine Institute, Memphis, \\ Tennessee, United States \\ ${ }^{3}$ Le Bonheur Children's Hospital, Memphis, Tennessee, United States \\ ${ }^{4}$ Department of Surgery, St. Jude Children's Research Hospital, \\ Memphis, Tennessee, United States \\ ${ }^{5}$ Department of Radiological Sciences, St. Jude Children's Research \\ Hospital, Memphis, Tennessee, United States \\ 6 Department of Epidemiology and Cancer Control, St. Jude Children's \\ Research Hospital, Memphis, Tennessee, United States
}

J Neurol Surg Rep 2013;74:96-100.

\author{
Gregory T. Armstrong $^{6} \quad$ L. Madison Michael II,2
}

\author{
Abstract \\ Keywords \\ - mature teratoma \\ - petrous \\ - temporal bone \\ - cerebellopontine \\ angle \\ - resection \\ - child \\ - pediatric
}

Address for correspondence Paul Klimo, Jr., MD, MPH, SemmesMurphey Neurologic \& Spine Institute, 6325 Humphreys Blvd., Memphis, TN 38120, United States

(e-mail: pklimo@semmes-murphey.com).

\section{Introduction}

Intracranial germ cell tumors are frequently diagnosed in children and typically arise from midline locations such as the suprasellar and pineal regions. ${ }^{1-3}$ Advancements in neuropathology, neuroradiology, and microsurgical techniques have allowed for improved diagnosis and treatment of pediatric teratomas. In this paper, the authors present a case of an 11-year-old girl with 12 months of progressive leftsided facial weakness. Imaging revealed a large temporal bone mass with extension into the cerebellopontine angle. A transtemporal approach was undertaken, and the pathology was consistent with a mature teratoma. To the authors' knowledge, this is the first case report of a mature teratoma originating in the petrous bone with extension into the cerebellopontine angle. received

February 5, 2013 accepted after revision May 20, 2013 published online July 23, 2013
DOI http://dx.doi.org/ $10.1055 / \mathrm{s}-0033-1349203$. ISSN 2193-6358.

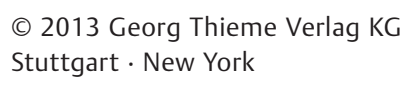

License terms

(®) $\Theta \circledast$ 


\section{Case Report}

\section{History and Examination}

A previously healthy 11 -year-old girl began experiencing leftsided facial weakness 12 months before presentation; her parents noted obvious asymmetry over the last 3 months along with daily headache. Past medical history was notable for congenital unilateral deafness (left-sided), but no previous imaging or further evaluation of this deficit had been pursued. Neurologic examination revealed multiple mid to lower cranial nerve deficits. She had limited abduction of the left eye, a House-Brackmann grade V/VI left-sided facial palsy, absent elevation of the left soft palate, and deviation of the tongue to the left. The patient had profound hearing loss in the left ear on audiologic evaluation. Results of motor, sensory, cerebellar, and gait testing were normal.

\section{Imaging}

Computed tomography (CT) demonstrated a multiloculated lesion expanding the labyrinthine structures in the left petrous temporal bone including the vestibule, semicircular canals, and cochlea, with extension to the left cerebellopontine angle via the expanded left internal auditory canal (-Fig. 1). There was no overt osseous destruction. A punctate intralesional calcification and at least two small foci of fat were evident. There was significant mass effect on the left pons, middle cerebellar peduncle, and cerebellum. Magnetic resonance (MR) imaging at 3-Tesla (Magnetom Trio, Siemens, Erlangen, Germany) confirmed these features and demonstrated a cerebrospinal fluid (CSF) signal intensity, diffusionrestricted component that was consistent with epidermoid cyst in the left prepontine cistern and compression of the left fifth cranial nerve between the cyst and the rim-enhancing multicystic tumor component (-Fig. 2). Dynamic susceptibility contrast (DSC) MR perfusion imaging demonstrated low

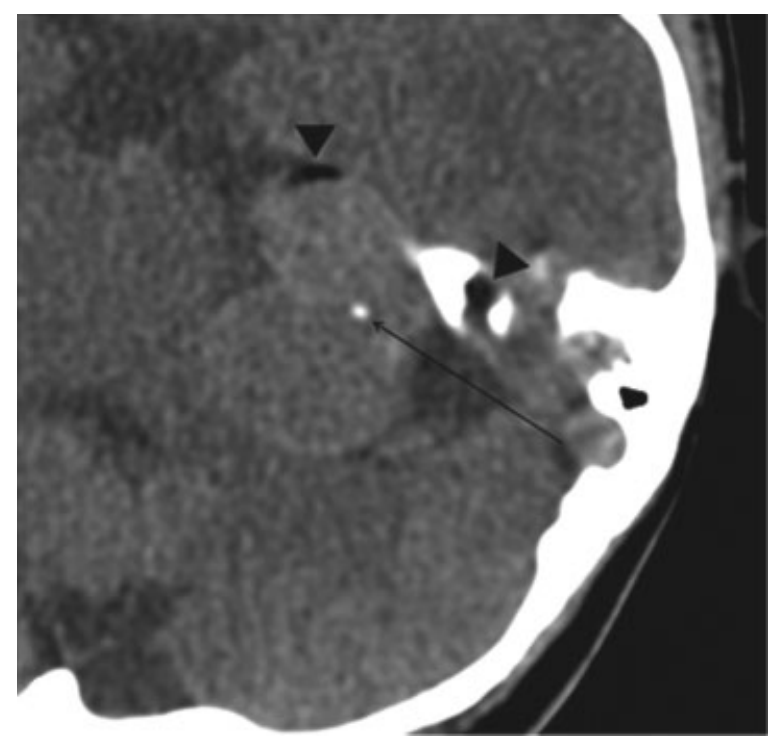

Fig. 1 Noncontrast axial computed tomography demonstrating a multiloculated lesion in the left cerebellopontine angle with punctate intralesional calcification (black arrow) and at least two small foci of fat at the periphery of the cysts (arrowheads).

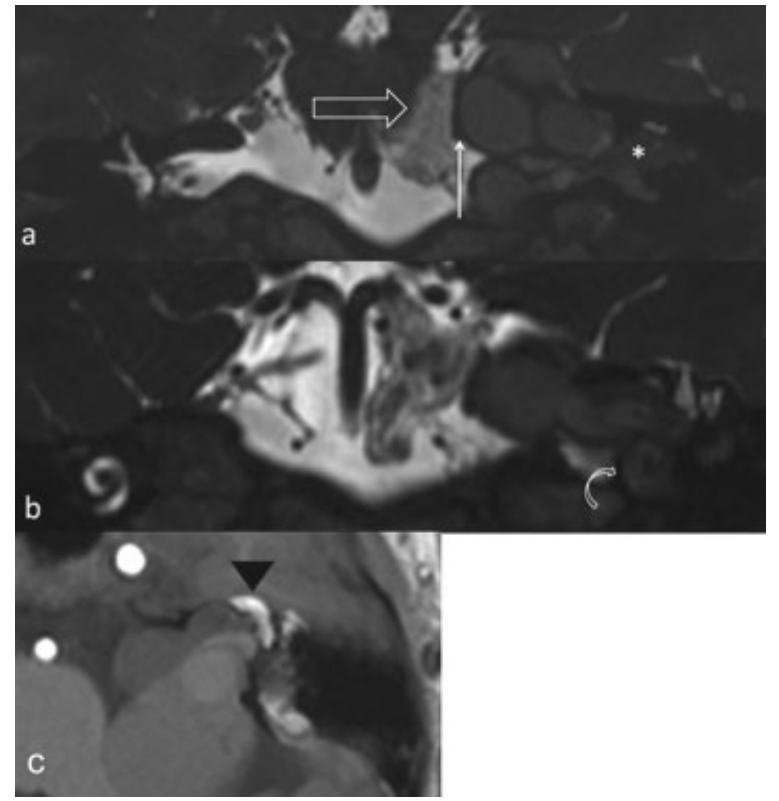

Fig. 2 (a, b) Coronal constructive interference in steady state (CISS) magnetic resonance imaging (MRI) demonstrating expansion of the left internal auditory canal, vestibule, semicircular canals $\left({ }^{*}\right)$ and cochlea (curved arrow). (c) Axial T1-weighted MRI without contrast demonstrating intralesional fat (arrowhead) and rim-enhancing, internally complex cystic components, with a more solid or microcystic appearance in the expanded labyrinthine structures.

lesional cerebral blood flow and blood volume (-Fig. 3). Single-voxel MR spectroscopy demonstrated a lone large Nacetyl L-aspartate (NAA) peak within a dominant, apparently proteinaceous cystic component, with no significant choline or creatine peaks (-Fig. 4).

\section{Operation}

The patient was placed in the supine position and her head was rotated toward the right shoulder. A "C"-shaped retroauricular incision was made. The skin flap was rotated anteriorly, and transection of the external auditory canal was performed. Immediately upon drilling the outer cortex of the mastoid portion of the temporal bone, tumor was encountered. A formal mastoidectomy was completed, although the tumor had skeletonized most of the soft tissue structures of the temporal bone. Stimulation of the labyrinthine and mastoid segments of the facial nerve was not successful even at $1.0 \mathrm{~mA}$. Tumor was easily removed from the petrous portion of the internal carotid artery and the internal auditory canal. After removal of the tumor within the extradural temporal bone, the presigmoid dura was widely opened, and the posterior fossa contents were exposed. Two large cysts were incised and drained, which promoted relaxation of the adjacent neural structures. The remainder of the intradural tumor was solid.

The facial nerve was identified at the level of the brainstem and stimulation was absent at $1.0 \mathrm{~mA}$. The seventh nerve was intimately involved with the tumor at the level of the porus acusticus, such that gross total resection without sacrifice seemed unlikely. As stimulation of the facial nerve was 


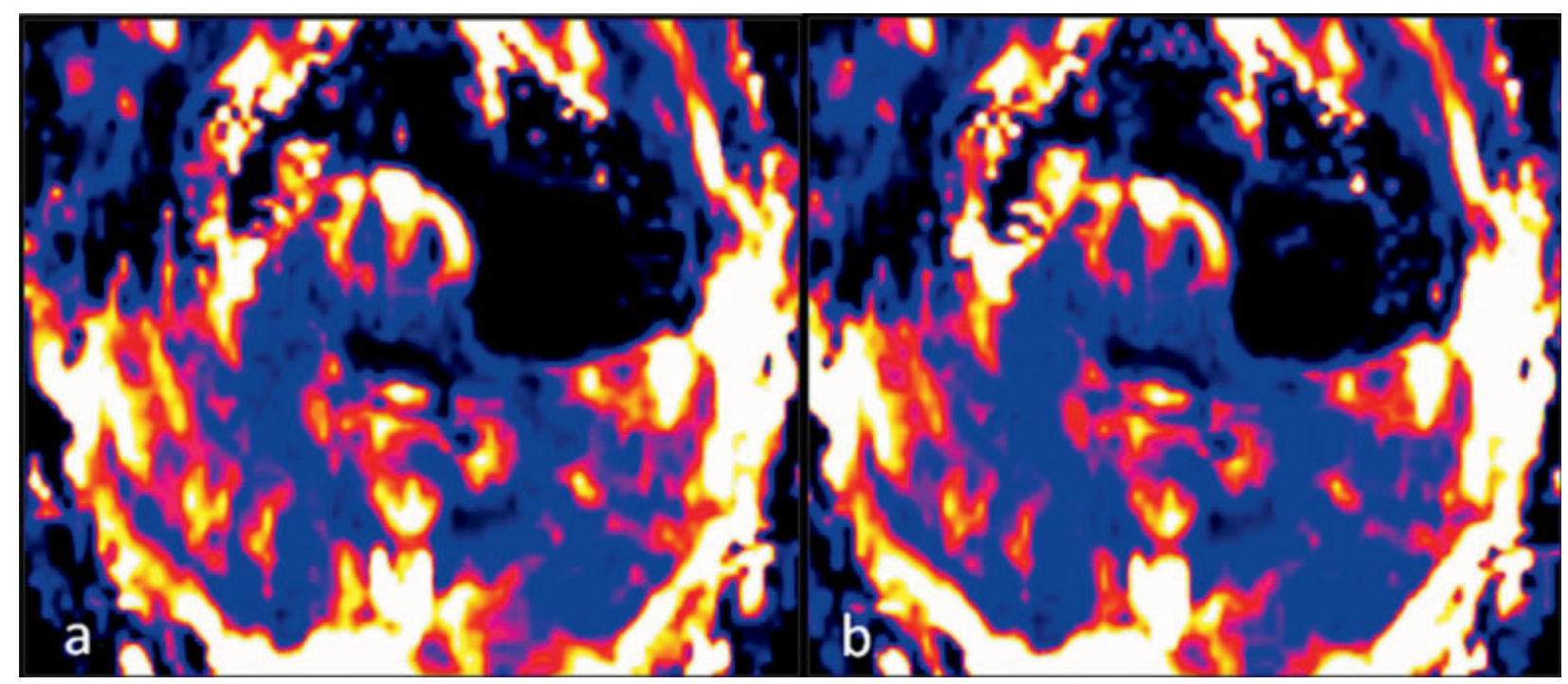

Fig. 3 Dynamic susceptibility contrast magnetic resonance (MR) perfusion imaging demonstrating low lesional cerebral blood flow and cerebral blood volume.

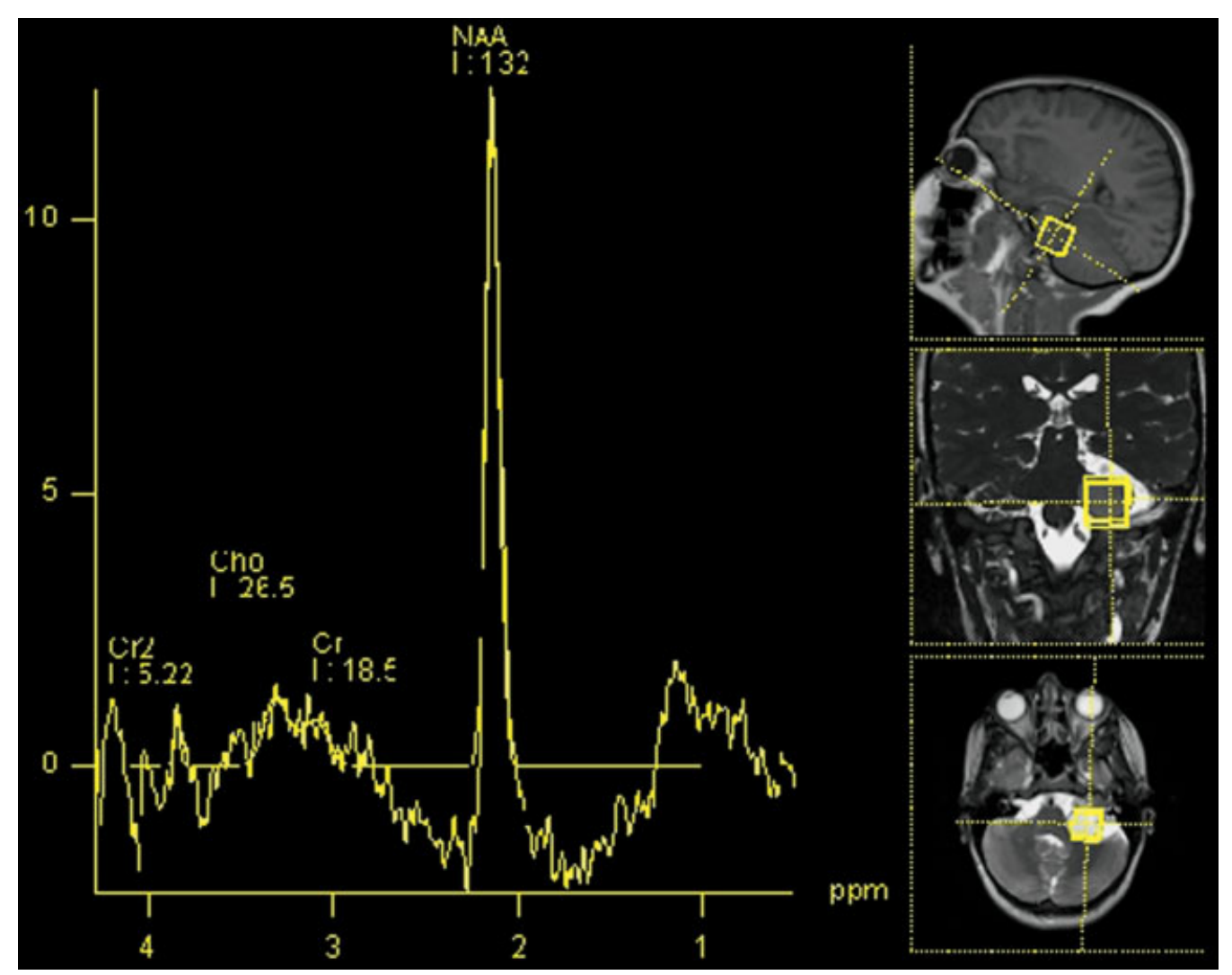

Fig. 4 Single-voxel magnetic resonance (MR) spectroscopy demonstrating a lone N-acetyl L-aspartate (NAA) peak in the dominant, proteinaceous-appearing, cystic component.

unsuccessful, the decision was made to transect the nerve. The tumor was somewhat adherent to the brainstem, but was successfully removed from it. Superiorly, the fifth nerve was associated with the tumor but was successfully dissected off the capsule. The sixth nerve was visualized medially and remained intact under a layer of arachnoid. The lower cranial nerves were visualized inferiorly. Intraoperative examination indicated that a gross total resection was achieved. The expanded cochlea was not thought to represent neoplasm. Adipose tissue was inserted into the eustachian tube to prevent leakage of CSF. Adipose tissue was also placed within the temporal bone defect, and the posterior aspect of the temporalis muscle was mobilized and placed over fat. 


\section{Pathology}

The lesion consisted of a complex and disorganized mixture of mature cartilage, bone, fat, smooth muscle, collagenous connective tissue, and various kinds of epithelial-lined glands and small cysts. There was a separate fragment of keratinous debris. There was no evidence of immature or malignant components. These findings were consistent with a mature teratoma.

\section{Postoperative Imaging}

Postoperative imaging demonstrated resection of all intracranial lesional components, residual deformity of the left fifth cranial nerve cisternal segment, resolution of mass effect on the cerebellum and brainstem, and stable signal abnormality within the residual expanded cochlea ( - Fig. 5). Follow-up MR imaging 24 months later demonstrated these findings to be stable.

\section{Postoperative Course}

The patient's neurologic examination remained stable with the exception of the expected complete facial paralysis. She remained in the hospital for 4 days and was discharged home. Prior to discharge, she received implantation of a gold weight in the left eyelid to promote closure. The patient did experience an episode of aseptic meningitis that responded to the administration of steroids. She returned 3 months after the operation and underwent a hypoglossal-to-facial nerve anastomosis. At her 1-year follow-up, her facial function had improved to a House-Brackmann grade V/VI facial palsy.

\section{Discussion}

Intracranial germ cell tumors can generally be divided into two categories: germinomas and tumors derived from toti-

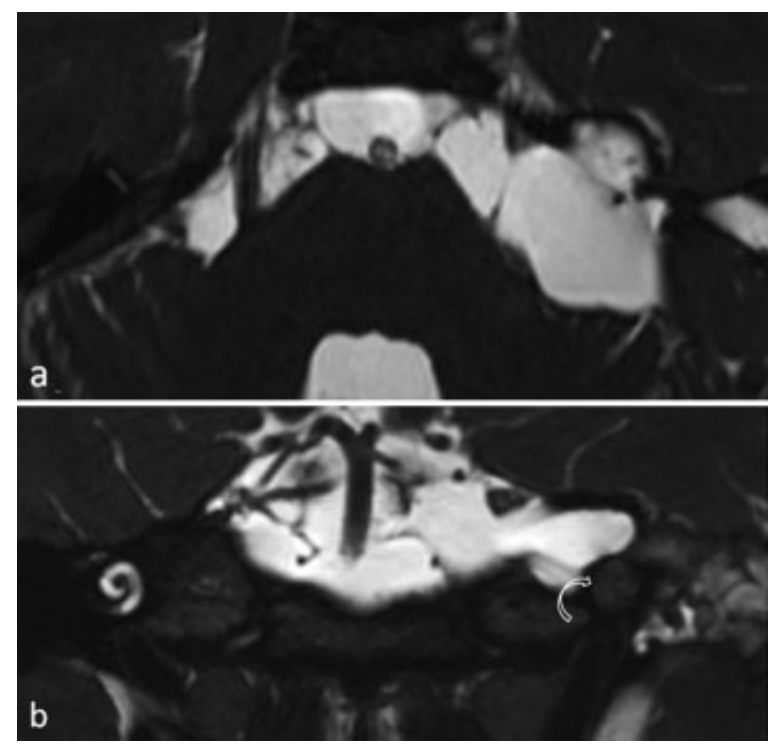

Fig. 5 Postoperative follow-up axial (a) and coronal (b) coronal constructive interference in steady state (CISS) magnetic resonance (MR) images demonstrating complete resection of the intracranial tumor components with stable signal abnormality in the residual expanded cochlea (curved arrow). Mass effect on the brainstem, cerebellum, and fifth cranial nerve has resolved. potential germ cells (also called nongerminomatous germ cell tumors). Nongerminomatous germ cell tumors have a wide spectrum of differentiation, with teratomas representing the most differentiated of these tumors. Mature teratomas contain fully differentiated tissue from all three primary germinal layers. Intracranial germ cell tumors represent $7 \%$ of intracranial tumors in the West and up to $15 \%$ in Japan. ${ }^{4,5}$ Mature teratomas comprise 18 to $20 \%$ of germ cell tumors. ${ }^{4}$ Most occur in the midline or paramidline locations, such as the pineal region and sellar/suprasellar area. Lateral locations for intracranial teratomas are exceptional. There have been cases reported of teratomas involving the ventricular system, Sylvian fissure, basal ganglia, pituitary fossa, cavernous sinus, middle ear space, petrous portion of the temporal bone, and middle cranial fossa. ${ }^{6-13}$ To our knowledge, this is the first case report of an intracranial teratoma involving the temporal bone with extension into the cerebellopontine angle. Li et al ${ }^{14}$ recently published their case of a 4-year-old girl who presented with fevers, headache, and purulent drainage from her ear. Imaging revealed a 4-cm lesion occupying the middle fossa with erosion of the petrous bone. Complete resection was achieved with an infratemporal epidural approach.

Pediatric intracranial germ cell tumors present at an average patient age of 10 to 12 years with variable presentations depending on location. ${ }^{1}$ Despite the unusual location in this case, foci of lesional calcification and fat on imaging are highly suggestive of the diagnosis, as in mature teratomas elsewhere in the body. As expected for a benign hypovascular tumor, the tumor was hypoperfused at MR perfusion imaging. Interestingly, NAA, though not generally found in high concentration outside brain tissues, was elevated in a proteinaceous-appearing cystic lesional component in this case and has also been found to be elevated in the cysts of serous ovarian tumors. ${ }^{15}$

Resection of teratomas is necessary to prevent malignant transformation ${ }^{16}$ and to obtain enough tissue to confirm the diagnosis because it is not uncommon for germ cell tumors to have mixed pathology. Complete microsurgical resection is the only curative treatment for pure teratomas. In the series by Matsutani et al, ${ }^{5}$ more than $90 \%$ of patients with pure teratomas who underwent a resection survived at least 10 years. Although chemotherapy and conventional irradiation are effective forms of treatment for germinoma and nongerminomatous germ cell tumors, they have no effect on teratomas. Radiosurgery, however, may have a role. Chiu et a ${ }^{17}$ described three patients with recurrent mature teratomas treated with marginal doses of 12 to 17 Gy. Tumor volume reduction ranged from $48 \%$ to $89 \%$, with no evidence of further tumor progression or radiosurgery-related morbidity.

\section{Conclusion}

As in our case, when a mature teratoma is known or highly suspected, complete resection must be the goal and will thus determine the surgical approach. Despite the involvement of multiple intracranial compartments, all efforts should be undertaken to completely visualize the lesion to maximize 
100 Petrous and Cerebellopontine Angle Mature Teratoma Khan et al.

the chance for surgical success. In this case, the transtemporal corridor provided excellent access to ensure that the utmost removal of tumor was accomplished.

\section{References}

1 Echevarría ME, Fangusaro J, Goldman S. Pediatric central nervous system germ cell tumors: a review. Oncologist 2008;13(6):690699

2 Merchut MP, Biller J, Ghobrial M, Fine M. Adult intrasellar teratoid tumor. J Clin Neuroophthalmol 1986;6(3):175-180

3 Nishioka H, Ito H, Haraoka J, Akada K. Immature teratoma originating from the pituitary gland: case report. Neurosurgery 1999;44(3):644-647, discussion 647-648

4 Noudel R, Vinchon M, Dhellemmes P, Litré CF, Rousseaux P. Intracranial teratomas in children: the role and timing of surgical removal. J Neurosurg Pediatr 2008;2(5):331-338

5 Matsutani M, Sano K, Takakura K, et al. Primary intracranial germ cell tumors: a clinical analysis of 153 histologically verified cases. J Neurosurg 1997;86(3):446-455

6 Drapkin AJ, Rose WS, Pellmar MB. Mature teratoma in the fourth ventricle of an adult: case report and review of the literature. Neurosurgery 1987;21(3):404-410

7 Muzumdar D, Goel A, Desai K, Shenoy A. Mature teratoma arising from the sella-case report. Neurol Med Chir (Tokyo) 2001;41 (7):356-359
8 Ng HK, Poon WS, Chan YL. Basal ganglia teratomas: report of three cases. Aust N Z J Surg 1992;62(6):436-440

9 Nishigaya K, Ueno T, Satou E, Nukui H, Kobayashi M. Mature teratoma incidentally found in the sylvian fissure: a report of an autopsy case. Noshuyo Byori 1994;11(2):131-134

10 Phadke RS, Shenoy AS, Hosangadi A, Nadkarni TD. Mature teratoma arising from the middle cranial fossa. Ann Diagn Pathol 2004;8 (1):28-31

11 Roncaroli F, Scheithauer BW, Pires MM, Rodrigues AS, Pereira JR Mature teratoma of the middle ear. Otol Neurotol 2001;22(1): 76-78

12 Selçuki M, Attar A, Yüceer N, Tuna H, Cakiroğlu E. Mature teratoma of the lateral ventricle: report of two cases. Acta Neurochir (Wien) 1998;140(2):171-174

13 Tobias S, Valarezo J, Meir K, Umansky F. Giant cavernous sinus teratoma: a clinical example of a rare entity: case report. Neurosurgery 2001;48(6):1367-1370, discussion 1370-1371

14 Li ST, Zhong J, Zhu J. Surgical management of the petrous teratoma. ANZ J Surg 2009;79(9):653-654

15 Kolwijck E, Wevers RA, Engelke UF, et al. Ovarian cyst fluid of serous ovarian tumors contains large quantities of the brain amino acid N-acetylaspartate. PLoS ONE 2010;5(4):e10293

16 Hoffman HJ, Otsubo $\mathrm{H}$, Hendrick EB, et al. Intracranial germ-cell tumors in children. J Neurosurg 1991;74(4):545-551

17 Chiu CD, Chung WY, Pan DH, Wong TT, Shih YH, Lee LS. Gamma knife radiosurgery for intracranial mature teratoma-long-term results and review of literature. Surg Neurol 2006;65(4):343-351 